\title{
Gauribidanur Low-Frequency Solar Spectrograph
}

\author{
P. Kishore • C. Kathiravan · R. Ramesh • \\ M. Rajalingam • Indrajit V. Barve
}

Received: 5 November 2013 / Accepted: 16 April 2014 / Published online: 26 April 2014

(C) The Author(s) 2014. This article is published with open access at Springerlink.com

\begin{abstract}
A new radio spectrograph, dedicated to observe the Sun, has been recently commissioned by the Indian Institute of Astrophysics (IIA) at the Gauribidanur Radio Observatory, about $100 \mathrm{~km}$ North of Bangalore. The instrument, called the Gauribidanur Lowfrequency Solar Spectrograph (GLOSS), operates in the frequency range $\approx 40-440 \mathrm{MHz}$. Radio emission in this frequency range originates close to the Sun, typically in the radial distance range $r \approx 1.1-2.0 \mathrm{R}_{\odot}$. This article describes the characteristics of the GLOSS and the first results.
\end{abstract}

Keywords Instrumentation and data management - Radio bursts, dynamic spectrum · Radio bursts, association with flares $\cdot$ Magnetic fields, corona

\section{Introduction}

Observations with radio spectrographs help to understand transient activity such as flares and coronal mass ejections (CMEs) in the solar atmosphere through their associated radio bursts, which appear either as a patch of continuum or a drifting emission from high to low frequencies as a function of time. Although bursts can occur over a wide range of frequencies, $\approx 10 \mathrm{kHz}-10 \mathrm{GHz}$, in ground-based observations most of them are observed with good contrast at frequencies $<500 \mathrm{MHz}$ (Benz, 1993). Radio emission at different frequencies originates at different levels in the solar atmosphere because of the decrease in the electron density with increasing radial distance. The appearance of bursts also changes from one frequency to the other. To obtain data on the radio emission related to flares and CMEs, near-simultaneous observations over a wide range of frequencies are therefore required.

There are numerous radio antennas with distinct transmission/reception properties. Conventional dipole, Yagi-Uda antennas are limited to observations over a very narrow range of frequencies (Kraus, Marhefka, and Khan, 2006). It was pointed out by Rumsey (1957)

P. Kishore $(\varangle) \cdot$ C. Kathiravan · R. Ramesh · M. Rajalingam · I.V. Barve Indian Institute of Astrophysics, Bangalore, Karnataka, India e-mail: kishore@iiap.res.in 
that the geometry of a structure specified entirely by its angles will be ideal for operation over a wide range of frequencies. This is consistent with the Babinet principle, i.e. the impedance of a structure that is self-complementary is independent of frequency. The combined principle of angle-only specified geometry and self-complementary structures led to the development of Log-Periodic Structures (LPS). Their characteristics such as the input impedance, the Voltage Standing-Wave Ratio (VSWR), radiation pattern is a periodic function of the logarithm of the frequency. Initially, the LPS were designed as planar structures. But later they were modified by folding two planar structures to obtain a non-planar Log-Periodic Dipole Antenna (LPDA) (Duhamel and Isbell, 1957; Isbell, 1960). The elaborate mathematical treatment for designing a LPDA was later developed by Carrell (1961a). The LPDAs are the typical radio-frequency (RF) signalreceiving elements in low-frequency radio astronomy $(<1 \mathrm{GHz})$, particularly where observations over a wide range of frequencies are required (Erickson and Fisher, 1974; Boischot et al., 1980; Ebenezer et al., 2001; Ramesh et al., 2008; Benz et al., 2009; Maan et al., 2013).

Using LPDAs, we have recently constructed a new spectrograph capable of observing in the frequency range $\approx 40-440 \mathrm{MHz}$ at the Gauribidanur Radio Observatory ${ }^{1}$ to investigate 1) the U-type bursts in which the drift changes gradually from low to high frequencies some time after their initial drift from high to low frequencies. These bursts are interpreted as signatures of radio emission associated with closed magnetic-field lines in the solar atmosphere (Benz, 1993). 2) Type IV bursts (start frequency range $\approx 300-400 \mathrm{MHz}$ ) and their association with X-ray, microwave, white-light emissions, as well as their possible role as coronal magnetic-field probes (Aurass et al., 2003; Ramesh et al., 2013a). 3) Quasi-periodic type III radio bursts and their usefulness for coronal seismology (Mangeney and Pick, 1989; Aschwanden, Benz, and Montello, 1994; Ramesh et al., 2003, 2005). 4) The fundamental (F) harmonic $(\mathrm{H})$ type II bursts and their association with the flares and the CMEs (Claßen and Aurass, 2002; Gopalswamy et al., 2005; Nindos et al., 2008; Ramesh et al., 2010b; Magdalenić et al., 2010; Cho et al., 2013). 5) Type I bursts (occurrence frequency range $\approx 100-$ $400 \mathrm{MHz}$ range) and their association with CMEs (Kerdraon et al., 1983; Böhme, 1993; Chertok et al., 2001; Ramesh and Shanmugha Sundaram, 2001; Kathiravan, Ramesh, and Nataraj, 2007; Iwai et al., 2012). 6) Absorption bursts and their association with the onset of CMEs and coronal dimming (Ramesh and Sastry, 2000; Ramesh and Ebenezer, 2001).

7) Radio bursts associated with energy releases $\lesssim 10^{27}$ erg (Kundu et al., 1986; Kliem and Bastian, 1996; Ramesh et al., 2010a, 2013b; Saint-Hilaire, Vilmer, and Kerdraon, 2013; Iwai et al., 2013). This article describes the antenna array, the receiver system, and the first results.

\section{Design of the LPDA and Test Results}

Our idea was to design an LPDA that can work in the frequency range $40-440 \mathrm{MHz}$ with a gain of $\approx 7 \mathrm{~dB}$ (which is nearly the same as that of most of the commercial LPDAs), broad response/radiation pattern (beam) so that the Sun can be observed for a longer period of time without mechanically or electrically steering the antenna, and a VSWR $\lesssim 2$ so that there is a good impedance match and the incident signal on the antenna is maximally transmitted to the subsequent stages of the receiver system. We designed and fabricated the LPDAs at the Gauribidanur Radio Observatory following the standard procedure mentioned in the

\footnotetext{
${ }^{1}$ www.iiap.res.in/centers/radio.
} 


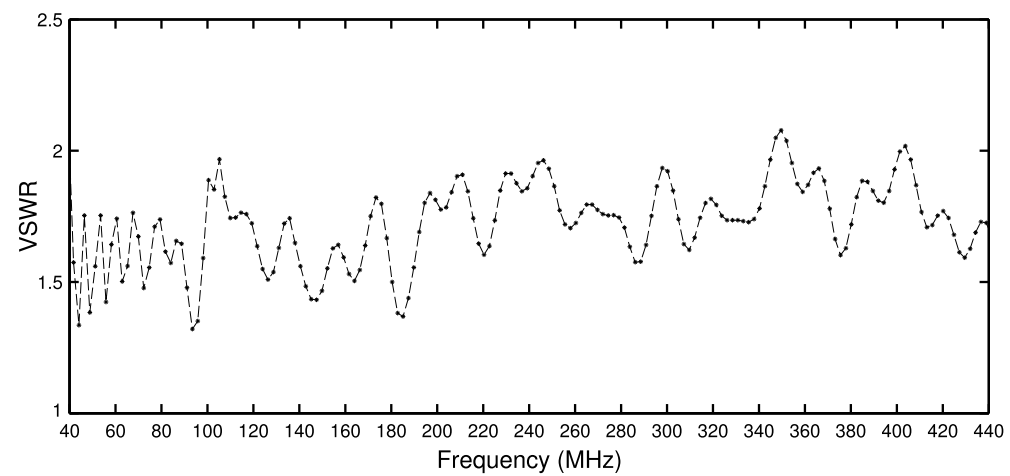

Figure 1 The Voltage Standing-Wave Ratio (VSWR) of one of the Log-Periodic Dipole Antennas (LPDAs) used in the GLOSS at different frequencies.

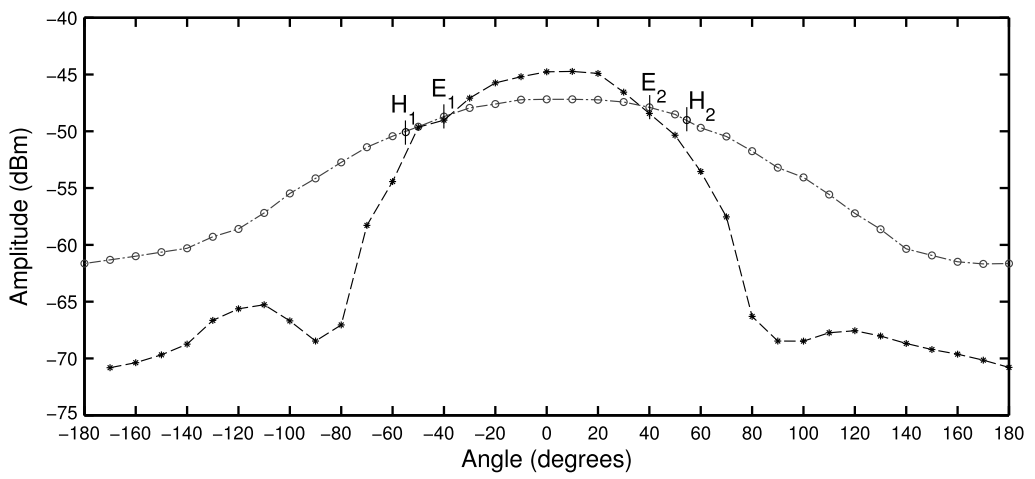

Figure 2 E-plane (asterisks) and H-plane (circles) patterns at $80 \mathrm{MHz}$ for one of the LPDAs used in the GLOSS. The angular separation corresponding to $\mathrm{E}_{1}-\mathrm{E}_{2}$ and $\mathrm{H}_{1}-\mathrm{H}_{2}$ indicates the HPBW along the E-plane and the H-plane, respectively.

literature (Carrell, 1961b; Wakabayashi et al., 1999; Balanis, 2005; Kraus, Marhefka, and Khan, 2006; Sasikumar Raja et al., 2013). Figure 1 shows the VSWR of the LPDAs used in the GLOSS. The values are $\lesssim 2$ over the entire frequency range $40-440 \mathrm{MHz}$. The far-field radiation pattern of the LPDAs designed for the GLOSS were measured in a manner similar to that described in Balanis (2005), Sasikumar Raja et al. (2013). Note that for any LPDA, response patterns are usually measured in two orthogonal directions: 1) in the direction of the arms of the LPDA (i.e. the E-plane) and 2) in the direction perpendicular to the arms of the LPDA (i.e. the H-plane). Figure 2 shows the E-plane and H-plane patterns at a typical frequency of $80 \mathrm{MHz}$. For the E-plane, the $3 \mathrm{~dB}$ or the half-power beam width (HPBW) of the pattern is $\approx 80^{\circ}$. The corresponding width for the H-plane pattern is $\approx 110^{\circ}$. The E-plane and H-plane widths were found to be approximately the same at different frequencies in the range of $40-440 \mathrm{MHz}$. The measured values of the HPBW along the E-plane $(\theta)$ and H-plane $(\phi)$ at $80 \mathrm{MHz}$ correspond to a solid angle of $\Omega=\theta \phi \approx 2.7 \mathrm{sr}$. From this we calculated the directional gain of the antenna, i.e. $G=10 \log _{10}(4 \pi / \Omega) \approx 6.7 \mathrm{dBi}$. The gain is approximately constant in the frequency range $40-440 \mathrm{MHz}$ (Figure 3 ). We calculated the effective collecting area from the gain using the relationship $A_{\mathrm{e}}=(G / 4 \pi) \lambda^{2} \approx 0.4 \lambda^{2}$. 


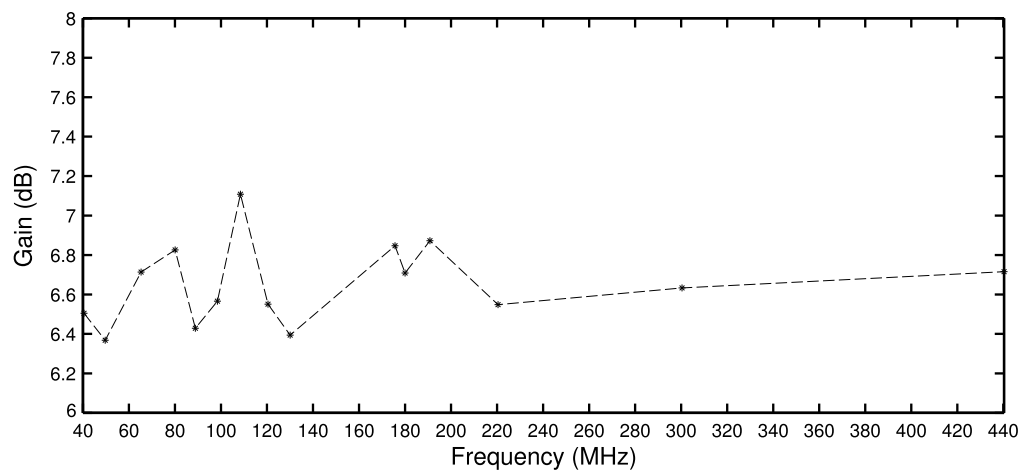

Figure 3 Gain of one of the LPDAs used in the GLOSS at different frequencies.

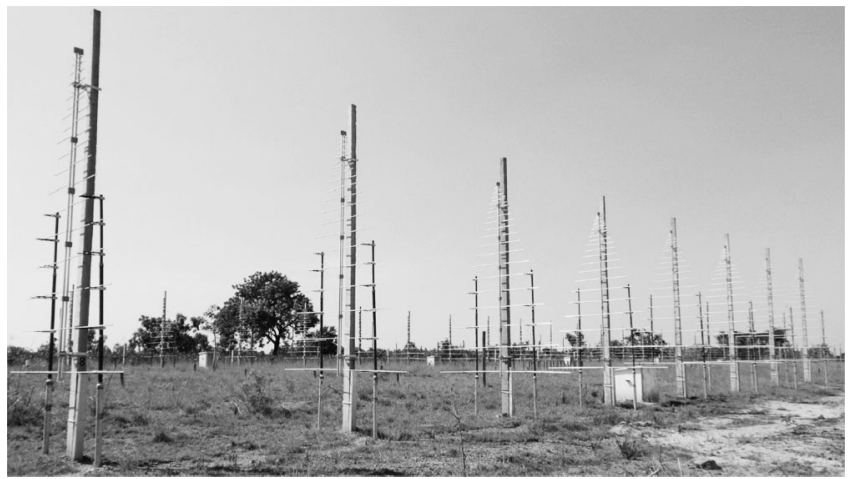

Figure 4 The GLOSS antenna system.

\section{Array Setup and Receiver System}

A group of eight similar LPDAs were mounted vertically pointing toward the local zenith $\left(\approx 77^{\circ} \mathrm{E} 14^{\circ} \mathrm{N}\right)$, and combined as an adding interferometer on a North-South baseline (Figure 4). The spacing between the adjacent LPDAs is $5 \mathrm{~m}$. The arms of all the LPDAs were oriented in the East-West direction. This implies that the E-plane of the all the LPDAs are along the East-West direction and the H-plane is along the North-South direction. The above arrangement results in a half-power beam width of $\approx 80^{\circ} \times 6^{\circ}$ (where the first value corresponds to the right ascension (RA) and the second one to the declination $(\delta)$ ) at a typical frequency of $80 \mathrm{MHz}$ for observations at the zenith. The output from each LPDA is passed through a high-pass filter with a $3 \mathrm{~dB}$ cut-off at $\approx 40 \mathrm{MHz}$ (Figure 5). This is to attenuate the spurious and unwanted RF signal at lower frequencies. The filtered signal is then amplified to $\approx 28 \mathrm{~dB}$ with a low-noise amplifier. This is called the pre-amplifier and has a uniform gain in the range $0.5-500 \mathrm{MHz}$ with a noise figure of $\approx 3 \mathrm{~dB}$. The amplified outputs from the LPDAs are combined using two-way power combiners and analog beamformer network circuits, i.e. coaxial cable delay-system, in three stages DSB I, DSB II, and DSB III (Figure 5). Note that because of the array geometry with respect to the declination of the Sun on any given day, there will be a geometrical phase difference, $\phi_{\mathrm{g}}=\frac{2 \pi}{\lambda} d \sin \theta$, between the RF signal incident on the LPDAs in the array; here $d$ is the distance between 


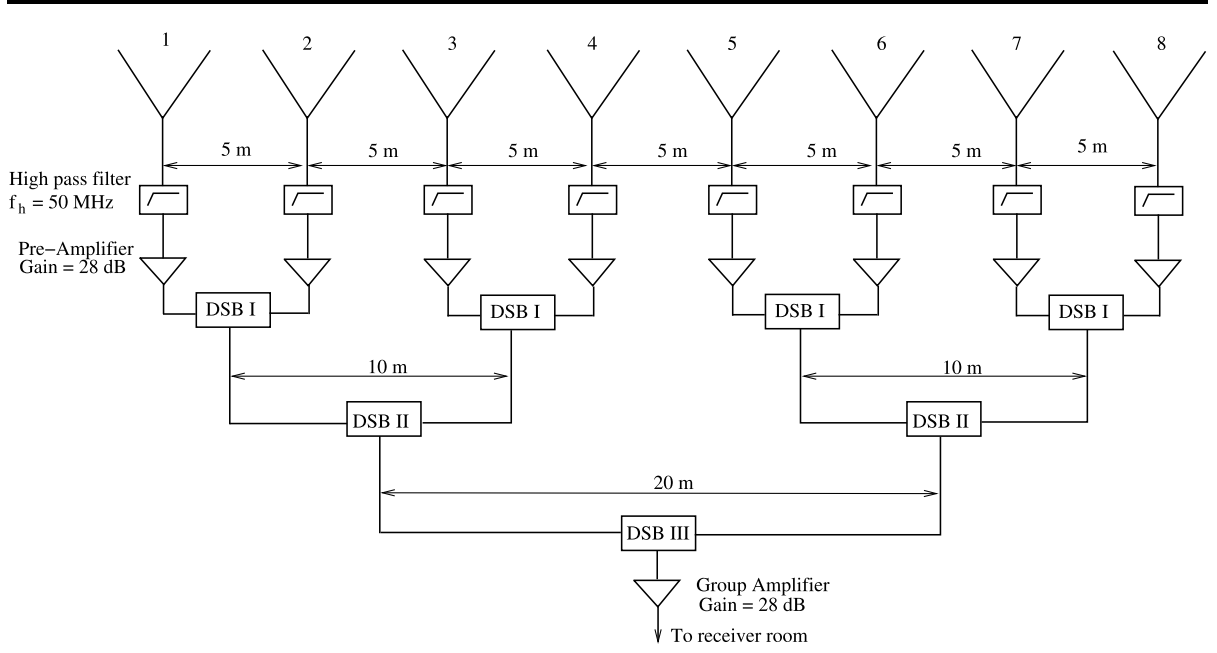

Figure 5 Radio frequency (RF) signal path from the individual LPDAs in the GLOSS.

the adjacent LPDAs or pairs of LPDAs, and $\theta$ is the zenith angle of the source in the NorthSouth direction (i.e. along the declination). This phase difference should be compensated for before the signals are combined. The analog beam-former network takes care of this. Each of them consists of coaxial cables of different lengths that can be selectively included in the RF signal-path via control signals transmitted from the receiver room (Landecker, 1984; Ramesh et al., 1998). These cables provide the instrumental phase $\phi_{\mathrm{i}}$ to compensate for $\phi_{\mathrm{g}}$ over the zenith angle range $-37^{\circ} \lesssim \theta \lesssim+9^{\circ}$. Note that the HPBW of the beam along the declination is $\approx 100^{\circ}$ (see Section 2 ). Since the local latitude at Gauribidanur is $\approx 14^{\circ}$ North, this spread in zenith angle covers the declination range $\delta=-23^{\circ}$ to $+23^{\circ}$ along which the Sun moves in a year. Care must be taken that the residual phase error $\Delta \phi=\phi_{\mathrm{g}}-\phi_{\mathrm{i}}$ is as small as possible. For GLOSS, the $\Delta \phi_{\mathrm{i}} \approx 12.5^{\circ}$ at highest operation frequency i.e. $440 \mathrm{MHz}$. The error is expected to be smaller at lower frequencies.

The output from the final beam-former DSB III is amplified to $\approx 28 \mathrm{~dB}$ in the group amplifier, similarly to the pre-amplifier mentioned above. The amplified output is transmitted to the receiver room $(\approx 200 \mathrm{~m}$ away) through a low-loss cable. The latter is buried about $\sim 1 \mathrm{~m}$ below the ground to minimize diurnal variations. The signal attenuation in this cable is $\approx 2 \mathrm{~dB}$ at the highest operating frequency of the GLOSS, i.e. $440 \mathrm{MHz}$. Note that the spectral observations of the solar radio bursts at low frequencies are usually carried out using only a single LPDA (Benz et al., 2009). The eight LPDAs in the GLOSS cover a comparatively larger effective collecting area $\left(A_{\mathrm{e}} \approx 3 \lambda^{2}\right)$ and better directivity, particularly along the declination. The lowest detectable flux density $\left(\Delta S_{\min }\right)$ with the GLOSS at $5 \sigma$ level is $\approx 0.5 \mathrm{sfu}$ ( $\mathrm{sfu}=$ solar flux unit $=10^{-22} \mathrm{Wm}^{-2} \mathrm{~Hz}^{-1}$ ) at $40 \mathrm{MHz}$ and $\approx 3 \mathrm{sfu}$ at $440 \mathrm{MHz}$. The comparatively larger $A_{\mathrm{e}}$ and hence the lower $\Delta S_{\min }$ of the GLOSS facilitates observations of radio bursts from the Sun with better contrast and strong cosmic radio sources for calibration purposes. The frequency dependence of $\Delta S_{\min }$ is due to the change in both the system temperature $T_{\text {sys }}$ (dominated by the background sky temperature at low frequencies) and the effective aperture $\left(A_{\mathrm{e}}\right)$ as a function of frequency.

In the receiver room the RF signal is passed through a high-pass filter followed by an amplifier similar to the antenna end, and then connected to a spectrum analyzer. We use the commercially available Agilent Spectrum Analyzer (Model E4401B, frequency range 
Table 1 Characteristics of the GLOSS.

\begin{tabular}{ll}
\hline Parameter & Value \\
\hline Frequency range & $\approx 40-440 \mathrm{MHz}$ \\
Number of frequency points & $\approx 401$ \\
Frequency resolution & $\approx 1 \mathrm{MHz}$ \\
Sweep time (40-440 MHz) & $\approx 250 \mathrm{~ms}$ \\
Total observation duration & $\approx 7 \mathrm{hr}$ \\
Local meridian & $\approx 06: 30 \mathrm{UT}$ \\
Effective collecting area & $\approx 3 \lambda^{2}$ \\
Lowest detectable flux density $(5 \sigma$ level $)$ & $\approx 0.5 \mathrm{sfu}(40 \mathrm{MHz})$ \\
& $\approx 3 \mathrm{sfu}(440 \mathrm{MHz})$ \\
Location & $\approx 77^{\circ} \mathrm{E} 14^{\circ} \mathrm{N}$ \\
\hline
\end{tabular}

$9 \mathrm{kHz}-1.5 \mathrm{GHz}$ ) to record the spectrum of solar radio bursts. The instrument is used in the continuous-sweep mode in the frequency range $\approx 40-440 \mathrm{MHz}$. The number of samples acquired in one sweep $(\approx 250 \mathrm{~ms})$ is 401 with a frequency resolution of $\approx 1 \mathrm{MHz}$. This sweep time is reasonable since the duration of the majority of solar radio bursts, particularly at low frequencies, is $\gtrsim 1 \mathrm{~s}$ (McLean, 1985). The interface to the computer is made with the commercially available GPIB-PCI card (Agilent 82350B). Data acquisition is carried out using Visual C++ (Ebenezer et al., 2007). Table 1 lists the characteristics of the GLOSS.

We stress that the spacing of $5 \mathrm{~m}$ between the adjacent LPDAs in the GLOSS is greater than the observation wavelengths at frequencies $\geq 60 \mathrm{MHz}$. Then, the array has grating lobes in the corresponding frequency range, i.e. $60-440 \mathrm{MHz}$ (Kraus, 1950). The separation between the main lobe and grating lobe is $\approx 8^{\circ}$ at $440 \mathrm{MHz}$ and $\approx 90^{\circ}$ at $60 \mathrm{MHz}$. However, any contribution to the GLOSS output from the possible presence of strong radio-wave emitting solar/cosmic sources that simultaneously occur at the location of the grating lobes as well as the main lobe in the sky is expected to be small because 1) the size of the Sun in the radio range increases with decreasing frequency. The average sizes are $\approx 0.6^{\circ}$ and $\approx 0.8^{\circ}$ at 440 and $60 \mathrm{MHz}$, respectively (Sheridan and McLean, 1985; Avignon et al., 1975). Compared with this, the grating lobes at the corresponding frequencies are far away, so that there will not be multiple lobes present on the Sun at the same time. 2) The flux densities of most radio bursts from the Sun are higher than those of radio emission from cosmic radio sources (Nelson, Sheridan, and Suzuki, 1985). Their temporal and spectral characteristics are different as well.

\section{Observations}

The GLOSS has been operating regularly for almost the past two years. The daily observing period is $\approx 2-11$ UT. Several interesting radio bursts have been observed since then. The HPBW of the GLOSS in the East-West direction $\left(\approx 80^{\circ}\right)$ is centered on the local meridian at Gauribidanur (see Section 3). This width corresponds to $\approx 5.5 \mathrm{hr}$ in time. But in practice we carry out observations for longer periods $(\approx 9 \mathrm{hr})$ since the solar radio bursts, particularly at low frequencies, are usually very intense (Nelson, Sheridan, and Suzuki, 1985). Figure 6 shows the background-subtracted GLOSS dynamic spectrum of a type III burst followed by an F-H type II radio burst observed on 17 November 2011 during the interval $\approx 07: 28-$ 07:31 UT. The frequency range corresponding to the $\mathrm{F}$ and $\mathrm{H}$ components of the type II 


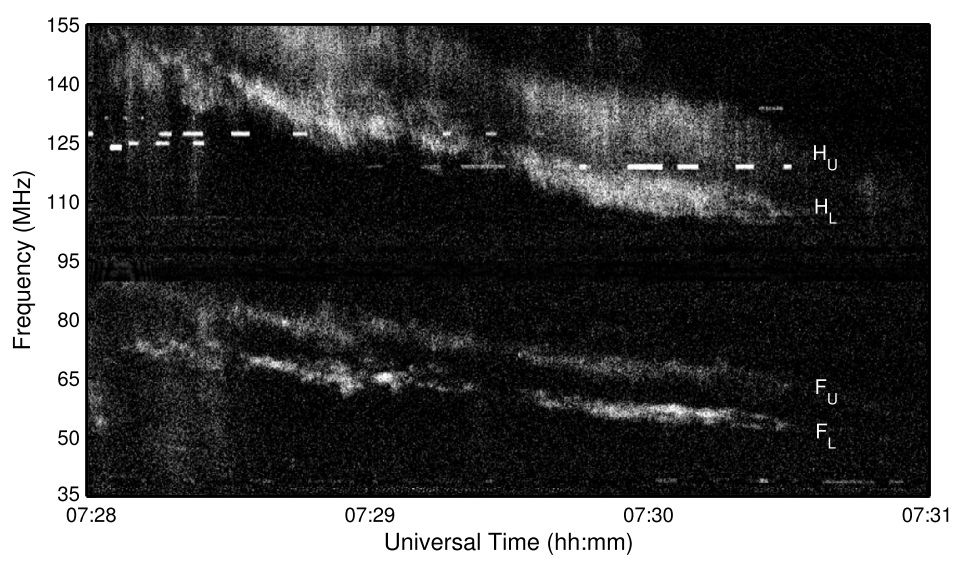

Figure 6 GLOSS observations of type III radio bursts followed by an F-H band-splitting type II burst on 17 November 2011. The horizontal dashed lines of intense emission near $125 \mathrm{MHz}$ are due to local radio frequency interference (RFI).

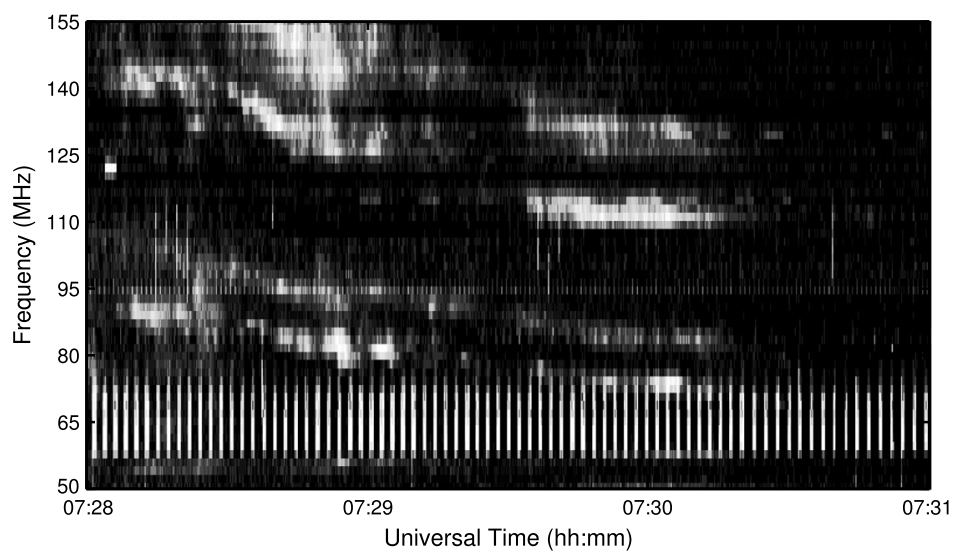

Figure 7 Same observations as in Figure 6, but with a single LPDA. The vertical stripes centered at $65 \mathrm{MHz}$ are instrumental artifacts.

burst in Figure 6 are $\approx 55-85 \mathrm{MHz}$, and $\approx 110-155 \mathrm{MHz}$. Both components show a splitband structure with distinguishable upper and lower bands, i.e. $\mathrm{F}_{\mathrm{U}}, \mathrm{F}_{\mathrm{L}}, \mathrm{H}_{\mathrm{U}}$, and $\mathrm{H}_{\mathrm{L}}$ (Nelson and Melrose, 1985). To observationally verify the effect of the comparatively larger $A_{\mathrm{e}}$ and hence lower $\Delta S_{\min }$ of GLOSS as compared with a single LPDA (see Section 3), we carried out simultaneous observations with the GLOSS and a single LPDA. The signal transmission and data acquisition in the two cases were similar. Figure 7 shows the observations of the same radio bursts as in Figure 6, but with a single LPDA. The enhanced contrast of the spectral features in GLOSS observations is clearly evident.

Figure 8 shows the spectral profile of the type II burst in Figure 6 at $\approx 07: 30$ UT. The total bandwidth of the $\mathrm{F}$ and $\mathrm{H}$ components, peak frequencies of $\mathrm{F}_{\mathrm{L}}$ and $\mathrm{H}_{\mathrm{L}}$ and $\mathrm{F}_{\mathrm{U}}$ and $\mathrm{H}_{\mathrm{U}}$ have a ratio of $\approx 1: 2$. The intensity of the harmonic component is higher than that of the fundamental component (Wild, Murray, and Rowe, 1954; Roberts, 1959). The event was associated with a C 6.0 class soft X-ray flare $(07: 16-07: 30 \mathrm{UT})$ with peak at $\approx 07: 27 \mathrm{UT}$ 


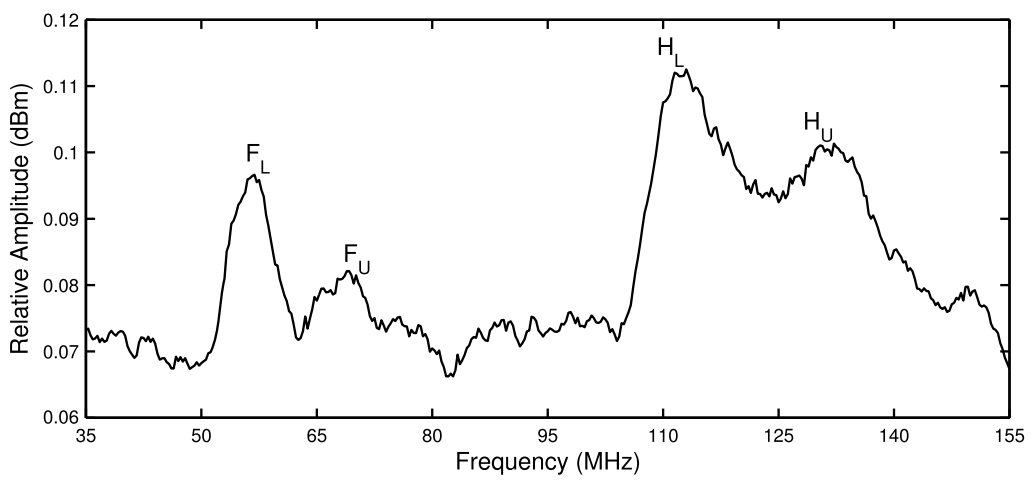

Figure 8 Spectral profile of the type II burst in Figure 6 at $\approx 07: 30$ UT.

and an SF-class H $\alpha$ flare (07:19-07:29 UT) with a peak at $\approx 07: 22$ UT from AR 11346 located at S19 E08. ${ }^{2}$ The Large Angle and Spectrometric Coronagraph C2 (LASCO C2, Brueckner et al., 1995) onboard the orbiting Solar and Heliospheric Observatory (SOHO) observed a CME around the same time as this flare and radio burst. The CME was first noticed in the coronagraph field of view at $\approx 07: 48$ UT when its leading edge was at a radial distance $r \approx 2.6 \mathrm{R}_{\odot}$. Its central position angle and angular width were $\approx 81^{\circ}$ and $97^{\circ}$, respectively. ${ }^{3}$ The projected speed of the CME in the plane of the sky was $\approx 458 \mathrm{~km} \mathrm{~s}^{-1}$. It was a decelerating event $\left(\approx-38 \mathrm{~m} \mathrm{~s}^{-2}\right)$. The speed between the initial two height-time measurements was $\approx 580 \mathrm{~km} \mathrm{~s}^{-1}$.

Figure 9 shows the location of the fundamental component of the type II burst at $80 \mathrm{MHz}$, observed with the Gauribidanur Radio Heliograph (GRAPH, Ramesh et al., 1998; Ramesh, Subramanian, and Sastry, 1999; Ramesh, Sundara Rajan, and Sastry, 2006; Ramesh, 2011) around $\approx 07: 28: 30$ UT. An inspection of Figures 6 and 9 indicates that GRAPH observations correspond most likely to the upper frequency $\mathrm{F}_{\mathrm{U}}$ of the split-band structure in the fundamental component of the burst shown in Figure 6. The radial distance of the burst is $\approx 1.4 \mathrm{R}_{\odot}$, nearly the same as the extrapolated location of the leading edge of the CME (backward toward the Sun) around the same time, obtained using a quadratic fit to the height-time measurements. ${ }^{4}$ This spatio-temporal association between the type II burst and the CME is consistent with the statistical results reported recently by Ramesh et al. (2012).

We followed the methodology described in Cho et al. (2007) to calculate the magneticfield strength $(B)$ associated with the type II burst. Assuming the hybrid coronal electrondensity model of Vršnak, Magdalenić, and Zlobec (2004), we found that $B \approx 2.7-1.8 \mathrm{G}$ in the radial distance range $r \approx 1.4-1.6 \mathrm{R}_{\odot}$, nearly corresponding to the fundamental component in Figure 6. This density model gives a drift speed of $\approx 700 \mathrm{~km} \mathrm{~s}^{-1}$ for the type II burst, which agrees reasonably well with the estimated speed of the CME close to the Sun using its height-time measurements and deceleration mentioned above. We remark that the $B$ values are 1) about a factor of three higher than the estimates of the magnetic field in the 'undisturbed' corona in the similar height range (Ramesh, Kathiravan, and Satya Narayanan, 2011), and that they are 2) consistent with similar estimates for the 'disturbed' corona using

\footnotetext{
2 www.swpc.noaa.gov/ftpmenu/warehouse/2011.html.

${ }^{3}$ cdaw.gsfc.nasa.gov.

${ }^{4}$ cdaw.gsfc.nasa.gov/CME_list/UNIVERSAL/2011_11/.
} 
Figure 9 Composite of the radio heliogram (black contours) of the type II burst observed with the GRAPH at $80 \mathrm{MHz}$ on 17

November 2011 around $\approx 07: 28: 30 \mathrm{UT}$ and the SOHO/LASCO C2, SDO-AIA (193 $\AA$ ) images obtained around $\approx$ 08:00 UT on the same day. The peak brightness temperature $\left(T_{\mathrm{b}}\right)$ of the burst is $\approx 2.7 \times 10^{8} \mathrm{~K}$.

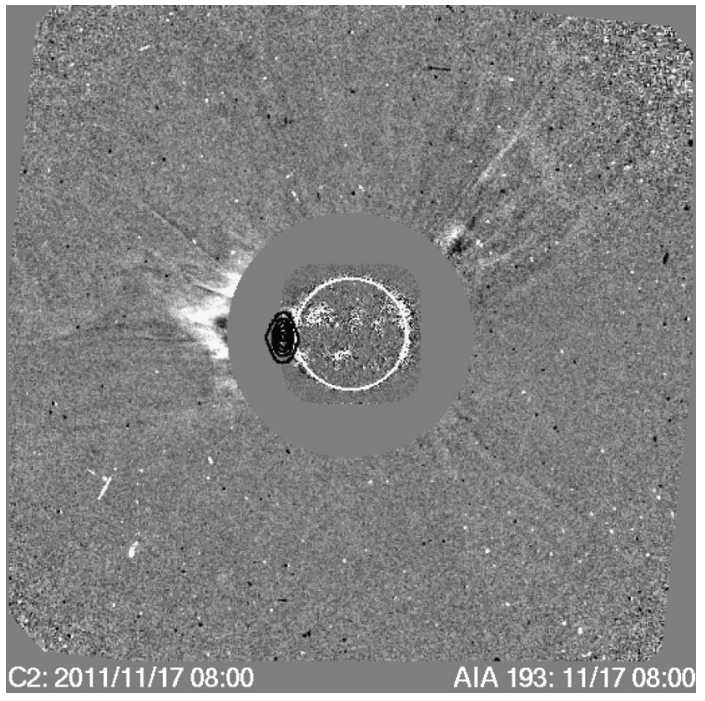

other types of radio bursts as tracers in the same height range (Dulk and McLean, 1978; Ramesh et al., 2003; Sasikumar Raja and Ramesh, 2013).

\section{Summary}

We have reported the commissioning of a new radio spectrograph for dedicated observations of the Sun from the Gauribidanur Radio Observatory near Bangalore, India and the first observations. The instrument operates in the frequency range $\approx 40-440 \mathrm{MHz}$. Daily solar observations with dedicated radio facilities such as the GLOSS and the GRAPH (for positional information of the bursts), particularly over a wide range of frequencies, are expected to be useful to understand the solar corona because 1) it has been suggested that reconnection sites at larger radial distances (higher altitudes in the solar atmosphere) are more prolific in coherent radio emission (Benz, 2004; Benz et al., 2005), 2) simulations indicate that the spectral characteristics of type III radio bursts are related to the coronal and electron heating conditions near their source region (Li, Cairns, and Robinson, 2009), 3) observations indicate that the Sun can be a source of significant radio emission even when the activity level is weak at other regions of the electromagnetic spectrum (Kliem and Bastian, 1996; Ramesh et al., 2010a). The scientific community will soon have free access to the daily observations of the facilities just discussed at our institute website.

Acknowledgements We thank the staff of the Gauribidanur Radio Observatory for their help in the fabrication, testing, and installation of the GLOSS antennas. The SOHO data are produced by a consortium of the Naval Research Laboratory (USA), Max-Planck-Institut für Aeronomie (Germany), Laboratoire d'Astronomie (France), and the University of Birmingham (UK). SOHO is a project of international cooperation between ESA and NASA. The SOHO-LASCO CME catalog is generated and maintained at the CDAW Data Center by NASA and the Catholic University of America in cooperation with the Naval Research Laboratory. The SDO/AIA data are courtesy of the NASA/SDO and the AIA science teams. We are grateful to the referee for his/her comments, which helped us to bring out the results more clearly.

Open Access This article is distributed under the terms of the Creative Commons Attribution License which permits any use, distribution, and reproduction in any medium, provided the original author(s) and the source are credited. 


\section{References}

Aschwanden, M.J., Benz, A.O., Montello, M.L.: 1994, Astrophys. J. 431, 432.

Aurass, H., Klein, K.-L., Zlotnik, E.-Y., Zaitsev, V.V.: 2003, Astron. Astrophys. 410, 1001.

Avignon, Y., Lantos, P., Palagi, F., Patriarchi, P.: 1975, Solar Phys. 45, 141.

Balanis, C.A.: 2005, Antenna Theory: Analysis and Design, Wiley, New Delhi.

Benz, A.O.: 1993, Plasma Astrophysics, Kluwer Academic, Dordrecht.

Benz, A.O.: 2004, In: Gary, D.E., Keller, C.U. (eds.) Solar and Space Weather Radiophysics: Current Status and Future Developments, Astrophys. Space Sci. Library 314, Kluwer Academic, Dordrecht, 203.

Benz, A.O., Monstein, C., Meyer, H., Manoharan, P.K., Ramesh, R., Altyntsev, A., Lara, A., Paez, J., Cho, K.-S.: 2009, Earth Moon Planets 104, 277.

Benz, A.O., Grigis, P.C., Csillaghy, A., Saint-Hilaire, P.: 2005, Solar Phys. 226, 141.

Böhme, A.: 1993, Solar Phys. 143, 151.

Boischot, A., Rosolen, C., Aubier, M.G., Daigne, G., Genova, F., Leblanc, Y., Lecacheux, A., de La Noe, J., Moller-Pedersen, B.: 1980, Icarus 43, 399.

Brueckner, G.E., Howard, R.A., Koomen, M.J., Korendyke, C.M., Michels, D.J., Moses, J.D., et al.: 1995, Solar Phys. 162, 357.

Carrell, R.L.: 1961a, Ph.D. thesis, Univ. Illinois.

Carrell, R.L.: 1961b, IRE Int. Conv. Rec. Part I, 61.

Chertok, I.M., Kahler, S., Aurass, H., Gnezdilov, A.A.: 2001, Solar Phys. 202, 337.

Cho, K.-S., Lee, J., Gary, D.E., Moon, Y.-J., Park, Y.D.: 2007, Astrophys. J. 665, 799.

Cho, K.-S., Gopalswamy, N., Kwon, R.-Y., Kim, R.-S., Yashiro, S.: 2013, Astrophys. J. 765, 148.

Claßen, H.T., Aurass, H.: 2002, Astron. Astrophys. 384, 109.

Duhamel, R.H., Isbell, D.E.: 1957, IRE Nat. Conv. Rec. Part I, 119.

Dulk, G.A., McLean, D.J.: 1978, Solar Phys. 57, 279.

Ebenezer, E., Ramesh, R., Subramanian, K.R., Sundara Rajan, M.S., Sastry, C.V.: 2001, Astron. Astrophys. 367, 1112.

Ebenezer, E., Subramanian, K.R., Ramesh, R., Sundara Rajan, M.S., Kathiravan, C.: 2007, Bull. Astron. Soc. India 35, 111.

Erickson, W.C., Fisher, J.R.: 1974, Radio Sci. 9, 387.

Gopalswamy, N., Aguilar-Rodriguez, E., Yashiro, S., Nunes, S., Kaiser, M.L., Howard, R.A.: 2005, J. Geophys. Res. 110, A12S07.

Isbell, D.E.: 1960, IRE Trans. Antennas Propag. AP-8, 260.

Iwai, K., Miyoshi, Y., Masuda, S., Shimojo, M., Shiota, D., Inoue, S., Tsuchiya, F., Morioka, A., Misawa, H.: 2012, Astrophys. J. 744, 167.

Iwai, K., Masuda, S., Miyoshi, Y., Tsuchiya, F., Morioka, A., Misawa, H.: 2013, Astrophys. J. Lett. 768, L2.

Kathiravan, C., Ramesh, R., Nataraj, H.S.: 2007, Astrophys. J. Lett. 656, L37.

Kerdraon, A., Pick, M., Trottet, G., Sawyer, C., Illing, R., Wagner, W., House, L.: 1983, Astrophys. J. Lett. 265, L19.

Kliem, B., Bastian, T.S.: 1996, In: Taylor, A.R., Paredes, J.M. (eds.) Radio Emission from the Stars and the Sun CS-93, Astron. Soc. Pac., San Francisco, 372.

Kraus, J.D.: 1950, Radio Astronomy, McGraw-Hill, New York.

Kraus, J.D., Marhefka, R.J., Khan, A.S.: 2006, Antennas for All Applications, Tata McGraw-Hill, New Delhi.

Kundu, M.R., Gergely, T.E., Szabo, A., Loiacono, R., White, S.M.: 1986, Astrophys. J. 308, 436.

Landecker, T.J.: 1984, IEEE Trans. Instrum. Meas. IM-33(2), 78.

Li, B., Cairns, I.H., Robinson, P.A.: 2009, J. Geophys. Res. 114, A02104.

Maan, Y., Deshpande, A.A., Chandrashekar, V., Chennamangalam, J., Raghavendra Rao, K.B., Somashekar, R., et al.: 2013, Astrophys. J. Suppl. 204, 12.

Magdalenić, J., Marqué, C., Zhukov, A.N., Vršnak, B., Z̃ic, T.: 2010, Astrophys. J. 718, 266.

Mangeney, A., Pick, M.: 1989, Astron. Astrophys. 224, 242.

McLean, D.J.: 1985, In: McLean, D.J., Labrum, N.R. (eds.) Solar Radiophysics, Cambridge University Press, Cambridge, 37.

Nelson, G.J., Melrose, D.B.: 1985, In: McLean, D.J., Labrum, N.R. (eds.) Solar Radiophysics, Cambridge University Press, Cambridge, 333.

Nelson, G.J., Sheridan, K.V., Suzuki, S.: 1985, In: McLean, D.J., Labrum, N.R. (eds.) Solar Radiophysics, Cambridge University Press, Cambridge, 113.

Nindos, A., Aurass, H., Klein, K.-L., Trottet, G.: 2008, Solar Phys. 253, 3.

Ramesh, R.: 2011, In: Choudhuri, A.R., Banerjee, D. (eds.) First Asia-Pacific Solar Physics Meeting CS 2 , Astron. Soc., India, 55.

Ramesh, R., Ebenezer, E.: 2001, Astrophys. J. Lett. 558, L141.

Ramesh, R., Kathiravan, C., Satya Narayanan, A.: 2011, Astrophys. J. 734, 39. 
Ramesh, R., Sastry, C.V.: 2000, Astron. Astrophys. 358, 749.

Ramesh, R., Shanmugha Sundaram, G.A.: 2001, Solar Phys. 202, 355.

Ramesh, R., Subramanian, K.R., Sastry, C.V.: 1999, Astron. Astrophys. Suppl. 139, 179.

Ramesh, R., Sundara Rajan, M.S., Sastry, C.V.: 2006, Exp. Astron. 21, 31.

Ramesh, R., Subramanian, K.R., Sundara Rajan, M.S., Sastry, C.V.: 1998, Solar Phys. 181, 439.

Ramesh, R., Kathiravan, C., Satya Narayanan, A., Ebenezer, E.: 2003, Astron. Astrophys. 400, 753.

Ramesh, R., Kathiravan, C., Satya Narayanan, A., Sastry, Ch.V., Udaya Shankar, N.: 2005, Astron. Astrophys. 431, 353.

Ramesh, R., Kathiravan, C., Sundara Rajan, M.S., Barve, I.V., Sastry, C.V.: 2008, Solar Phys. 253, 319.

Ramesh, R., Kathiravan, C., Barve, I.V., Beeharry, G.K., Rajasekara, G.N.: 2010a, Astrophys. J. Lett. 719, L41.

Ramesh, R., Kathiravan, C., Kartha, S.S., Gopalswamy, N.: 2010b, Astrophys. J. 712, 188.

Ramesh, R., Anna Lakshmi, M., Kathiravan, C., Gopalswamy, N., Umapathy, S.: 2012, Astrophys. J. 752, 107.

Ramesh, R., Kishore, P., Mulay, S.M., Barve, I.B., Kathiravan, C., Wang, T.J.: 2013a, Astrophys. J. 778, 30.

Ramesh, R., Sasikumar Raja, K., Kathiravan, C., Satya Narayanan, A.: 2013b, Astrophys. J. 762, 89.

Roberts, J.A.: 1959, Aust. J. Phys. 12, 327.

Rumsey, V.H.: 1957, IRE Nat. Conv. Rec. Part I, 114.

Saint-Hilaire, P., Vilmer, N., Kerdraon, A.: 2013, Astrophys. J. 762, 60.

Sasikumar Raja, K., Ramesh, R.: 2013, Astrophys. J. 775, 38.

Sasikumar Raja, K., Kathiravan, C., Ramesh, R., Rajalingam, M., Barve, I.V.: 2013, Astrophys. J. Suppl. 207, 2.

Sheridan, K.V., McLean, D.J.: 1985, In: McLean, D.J., Labrum, N.R. (eds.) Solar Radiophysics, Cambridge University Press, Cambridge, 443.

Vršnak, B., Magdalenić, J., Zlobec, P.: 2004, Astron. Astrophys. 413, 753.

Wakabayashi, R., Shimada, K., Kawakami, H., Sato, G.: 1999, IEEE Trans. Electromagn. Compat. $41(2), 93$.

Wild, J.P., Murray, J.D., Rowe, W.C.: 1954, Aust. J. Phys. 7, 439. 